\title{
THREE-BODY STABILITY CRITERIA
}

\section{J.R. DONNISON \\ Department of Mathematical and Computing Sciences, Goldsmiths College, University of London, New Cross, London SE14 6NW, UK}

Progress has been made in understanding the stability of hierarchical three-body systems where the third body moves on an approximately Keplerian orbit about the centre of mass of the binary, at a distance large compared to the binary separation. Harrington $(1968,1969)$ showed analytically that provided the third body was sufficiently distant from the binary no secular terms appeared in the semi-major axis and the system was stable. Harrington $(1972,1975,1977)$ established numerically a critical minimum separation distance (or period) for a stable system in terms of the masses, unaffected by the relative inclinations of the orbits, except for angles close to $90^{\circ}$. Most subsequent investigations have therefore used planar configurations. Graziani \& Black (1981), Black (1982) and Pendleton \& Black (1983) again using long-term integration of the orbits obtained a criterion for high and low mass binaries. Donnison \& Mikulskis $(1992,1994,1995)$ carried out numerical integrations on prograde, retrogade, planetary and stellar triple systems and found for prograde systems very good quantitative agreement with the $c^{2} H$ method. Eggleton \& Kieselva (1995) suggested a critical distance ratio approximation determined by the masses in the system. Systems with eccentric orbits are covered using the period ratio determined by Kepler's third law.

An analytic approach for determining the stability of coplanar three-body systems was outlined by Golubev $(1967,1968)$ and independently by Szebehely \& Zare (1977) and Marchal \& Saari (1975). This extends the toplogy of the zerovelocity surfaces of Hill to general coplanar three-body motion and compares $c^{2} H$ ( $c$ is the angular momentum, $H$ the energy of the system) for a particular system with the critical values at the collinear Lagrange points. This gives a sufficient condition for stability against exchange of the outer mass and the binary masses. A new analytic criterion was recently developed by Aarseth \& Mardling using chaos theory.

To compare the various criteria consider where all the masses are equal and both the inner and outer orbits are circular, then the critical distance ratio $\left(q_{\text {out }} / a_{\text {in }}\right)_{0}$ for the different criteria are shown below ( $q_{\text {out }}$ is the closest approach of the third mass, $M_{3}$, to the centre of mass of the binary and $a_{i n}$ is the semi-major axis of the binary orbit).

\begin{tabular}{|l|l|l|l|l|l|}
\hline Harrington & $\begin{array}{l}\text { Eggleton \& } \\
\text { Kieselva }\end{array}$ & $\begin{array}{l}\text { Aarseth \& } \\
\text { Mardling }\end{array}$ & $\begin{array}{l}\text { Szebehely } \\
\text { \& Zare }\end{array}$ & $\begin{array}{l}\text { Donnison \& } \\
\text { Mikulskis }\end{array}$ & $\begin{array}{l}\text { Graziani } \\
\text { \& Black }\end{array}$ \\
\hline 3.5 & 3.12 & 3.3 & 3.2 & 3.3 & 5.0 \\
\hline
\end{tabular}

Even for this critical case the various criteria give diverse results with the majority lying in the range $3.1 \leq\left(q_{\text {out }} / a_{i n}\right)_{0} \leq 3.5$, with one of 5 . For a more detailed comparison consider the criteria when $M_{1}>M_{2}$ and $M_{2}=M_{3}$, the orbits remaining circular. The separation into two predicted ranges is evident. The most 
Triple systems: solid circle data; + Eggleton et al; $x$ Aarseth et al : " Harrington; solid triangle Donnison et al.

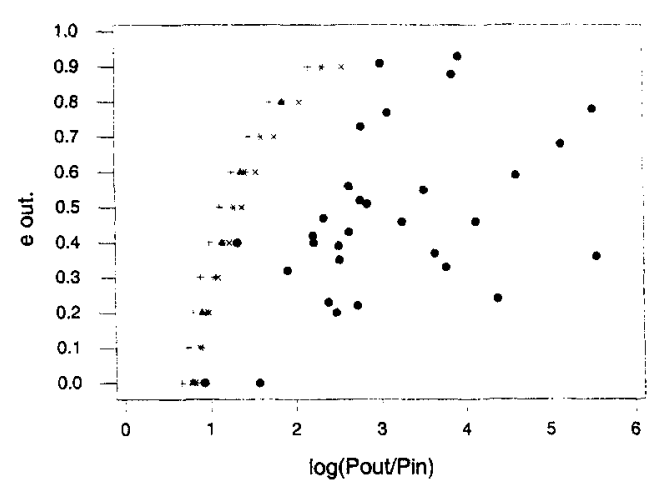

Fig. 1. $e_{\text {out }}$ against the $\log$ of the period ratio for triple systems (data from Fekel, 1981 and Chambliss, 1922). The equal mass criteria curves are shown superimposed.

likely reason for a variety of values is the definition and interpretation of stability in the manner of Laplace, that is the orbits of the masses are bounded so that the orbital elements (the semi-major axes and eccentricities) show no secular or large periodic variations during the time covered by the integrations. At high values of period ratio the criteria tend to converge, and most are also similar at small $e_{\text {out }}$ but significantly different from that of Black.

Multiple star systems can be divided into Trapezium systems with three or more stars whose separations are large and roughly equal and are dynamically unstable and hierarchical systems consisting of masses whose successive separations increase by large factors and can be described by a series of two-body motions. About $5-15 \%$ of all stellar systems are at least triple and nearly all of the observed multiple systems are hierarchical. Triple systems have recently been identified in open clusters. A plot of $e_{\text {out }}$ against the log of the period ratio for triple systems with spectroscopic binaries (spectroscopic-visual triples) the data being taken from Fekel (1981) and Chambliss (1992) are shown in fig.1 with the equal mass criteria curves shown superimposed. The real systems all lie to the right of the values given by the various criteria and should be stable.

\section{References}

Aarseth,S.J.\& Mardling,R.A.:1998 (personal communication).

Chambliss C.R.:1992, PASP, 104,663

Donnison J.R. \& Mikulskis D.F.:1992, MNRAS, 254, 21.

Donnison J.R. \& Mikulskis D.F.:1994, MNRAS, 266, 25.

Donnison J.R. \& Mikulskis D.F.:1995, MNRAS, 272, 1.

Eggleton P.P., Kiseleva L.G.:, 1995, ApJ, 455, 640.

Fekel F.C.:1981, ApJ, 246, 879

Golubev V.G.: 1967, Doklady Akad.Nauk SSSR, 12, 529.

Golubev V.G.: 1968, Doklady Akad. Nauk SSSR, 13, 373.

Harrington, R.S.:1968, AstrJ., 73, 190.

Harrington, R.S.:1969, Celest.Mech., 1, 200.

Harrington, R.S.:1972, Celest.Mech., 6, 322.

Harrington, R.S.:1975, Astr.J., 80, 1081.

Harrington, R.S.:1977, Astr.J., 82, 753.

Marchal,C. \& Saari,D.G.:1975, Celest.Mech.,12, 115.

Szebehely,V. \& Zare,K.:1977, A\& A, 58, 135. 